\title{
Addendum
}

\section{Dual Activation in Organocatalysis: Design of Tunable and Bifunctional Organocatalysts and Their Applications in Enantioselective Reactions}

Liang-Qiu Lu, Xiao-Lei An, Jia-Rong Chen, Wen-Jing Xiao* Synlett 2012, 23, 490.

In the advanced (e-first) version of this article the biographical sketches of the authors were missing. This has been corrected for the normal online and print versions. We apologize for this mistake. 